\title{
AN EFFICIENT IOT APPLICATION DEVELOPMENT BASED ON INTEGRATED IOT KNOWLEDGE MODULES
}

\author{
SeongYong Hong, Carson-Newman University, shong@cn.edu
}

\begin{abstract}
Internet of Things (IoT) has seen rapid growth in recent years, and this growth does not seem to be stopping any time soon. Therefore, since IoT has become a fast growing field, there are many opportunities for learning computer science that have opened up. IoT application development has taken on increasing importance as the rapid growth and complexity of computer science continue to expand. As the IoT continues its expansion into robotics, homes, hospitals, healthcare, businesses, government, global culture, and industries, the impact for computer science educators is amplified. An IoT application technology consists of web-enabled smart devices that use embedded systems, such as processors, sensors, and communication hardware, to collect, send, and act on data they acquire from their environments. But most computer science developers or students find it difficult to learn IoT technology because it requires different fields of knowledge of the computer technology environment. In this paper, we propose a method of efficient developing IoT application using integrated physical devices and mobile-based programming for computer science major colleges. In addition, we summarize our efforts, experiences, and reflections while teaching two offerings of an IoT concepts course.
\end{abstract}

Keywords: Internet of Things, IoT Knowledge, IoT Application, IoT Learner

\section{INTRODUCTION}

Recently, with ever increasing importance of IoT technology and business, there have been active researches on IoT learning throughout IoT development strategy. Internet of Things (IoT) has seen rapid growth in recent years, and this growth does not seem to be stopping any time soon. The global market for IoT technology, which consists of software, services, connectivity, and devices, reached $\$ 130$ billion in 2018. There are expected to be more than 64 billion IoT devices online and working actively by 2025. The Internet of Things is an ecosystem of physical objects that are connected and accessible through the internet. IoT enables devices to observe, understand, and analyze a situation or the surroundings without being dependent on human intervention. Billions of people around the world use smartphones, and that number is increasing rapidly every day. Therefore, it makes sense that mobile apps are the preferred channel for accessing IoT, due to the ease of developing them. Mobile is a far more flexible platform for transmitting data as well. With a single application on a device, IoT devices can be efficiently managed and monitored. Mobile applications play a significant role in enhancing the growth of IoT. IoT refers to the broad network of interconnected devices embedded with sensors and software for the purpose of transferring and sharing data over the internet. This allows data (information) collected from physical objects to be stored on dedicated servers or in the cloud. Analyzing this accumulated information makes it possible to achieve a safer, more convenient society by providing optimal device control and methods (Koreshoff, et al. 2013). An IoT smart home is a home that uses IoT to provide greater convenience and security. For example, home appliances can be connected to the internet and controlled through human interaction (voice, smartphone app), while attaching sensors enables automated control to deliver unprecedented levels of comfort and convenience (Abdullaha and Varolb, 2019). IoT Smart cities are cities that adopt IoT to achieve greater energy savings while minimizing environmental impact. The concept of smart cities has changed with the times, but at the moment, many cities are installing multiple sensors to acquire data on environmental conditions and consumer behavior as well as enable remote control of equipment and facilities to optimize operations and improve convenience for residents (Arasteh, et al. 2016). Connected cars based on IoT are cars that incorporate IoT to achieve safer, more comfortable driving. By acquiring and analyzing driving data obtained through onboard GPS, sensors, and cameras together with information on surrounding traffic it is possible to achieve safer driving control and provide optimal traffic guidance (Krasniqi and Hajrizi, 2016). IoT applications promise to bring immense value to our lives. With newer wireless networks, superior sensors, and revolutionary computing capabilities, the IoT could be the next frontier in the race for its share of the wallet. We can think of an intelligent device such as a traffic camera. The camera can monitor the streets for traffic congestion, accidents, weather conditions, and communicate this data to a common gateway. This gateway also receives data from other such cameras 
and relays the information further to a city-wide traffic monitoring system. IoT applications promise to bring immense value to our lives. Figure 1 explains the variety of application IoT in the real world. The IoT is a giant network of connected everything.

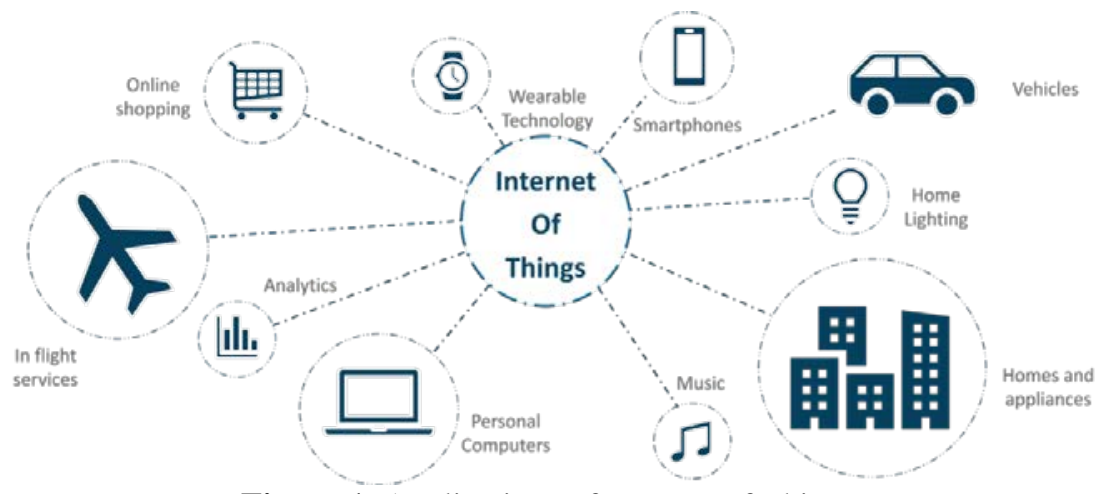

Figure 1. Applications of Internet of Things

An IoT application technology consists of web-enabled smart devices that use embedded systems, such as processors, sensors, and communication hardware, to collect, send, and act on data they acquire from their environments. But most computer science developers or students find it difficult to learn IoT technology because it requires different fields of knowledge of the computer technology environment (Ali, 2015). Therefore, in this paper, we propose a method of efficient developing IoT application using integrated physical devices and mobile-based programming for a computer science or information major college.

\section{RELATED WORKS}

IoT technology has played a very important and crucial role in transforming and changing the way we interact, communicate, and function in today's day and age. The internet of things is a system of interrelated computing devices, mechanical and digital machines, objects, animals, or people that are provided with unique identifiers (UIDs) and the ability to transfer data over a network without requiring human-to-human or human-to-computer interaction (Atzori, et al. 2010).

\section{Case Studies of IoT System Development}

The basic elements of the IoT are devices that gather data. Broadly speaking, they are internet-connected devices, so they each have an IP address. They range in complexity from autonomous vehicles that haul products around factory floors to simple sensors that monitor the temperature in buildings (Monnier, 2013). They also include personal devices like fitness trackers that monitor the number of steps individuals take each day. To make that data useful it needs to be collected, processed, filtered, and analyzed, each of which can be handled in a variety of ways. Collecting the data is done by transmitting it from the devices to a gathering point. Moving the data can be done wirelessly using a range of technologies or on wired networks (De La Concepcion, et al. 2014). The data can be sent over the internet to a data center or a cloud that has storage and compute power or the transfer can be staged, with intermediary devices aggregating the data before sending it along. Processing the data can take place in data centers or clouds, but sometimes that is not an option. In the case of critical devices such as shutoffs in industrial settings, the delay of sending data from the device to a remote data center is too great. The round-trip time for sending data, processing it, analyzing it, and returning instructions can take too long. In such cases, sensor computing can come into play, where a smart sensor device can aggregate data, analyze it, and fashion responses if necessary, all within relatively close physical distance, thereby reducing delay. Sensor devices also have upstream connectivity for sending data to be further processed and stored (Díaz Pardo, 2014). Essentially, anything that is capable of gathering some information about the physical world and sending it back to the system can participate in the IoT system. For example, smart home appliances, RFID tags, and industrial sensors are a few examples (Tan, et al. 2018). These sensors can monitor a range of factors including temperature and pressure in industrial systems, the status of critical parts in machinery, patient vital signs, and use of water and electricity, among many, many other possibilities (Sivaraman, et al. 2015). Entire factory robots can be considered IoT devices, as can autonomous vehicles that move products around industrial settings and 
warehouses (Deshpande, et al. 2016). Other examples include smart clothing, fitness wearables and home security systems. There are also more generic devices, like the Raspberry Pi or Arduino, which let us build our own IoT endpoints. Even though we might think of our smartphone as a pocket-sized computer, it may well also be beaming data about our location and behavior to back-end services in very IoT-like ways (Fernández and Fraga, 2018).

\section{Case Studies of IoT App Development}

There are many platforms that we can utilize to develop our IoT application. Mobile apps will be a point of connection for many IoT devices. In this part, we will present literature that reviews case studies of IoT app development.

Yeole, et al. (2015) studied a smartphone control robot using ATMEGA328 Microcontroller. In this research, they have designed a robot that can be controlled using an application running on an android phone. It sends control command via Bluetooth which has certain features like controlling the speed of the motor, sensing, and sharing the information with the phone about the direction and distance of the robot from the nearest obstacle. Pahuja and Kumar (2014) researched and tested an Android mobile phone control Bluetooth robot using 8051 microcontrollers. According to this study, Android Bluetooth enables phones and Bluetooth module via HC-06 and communication among Bluetooth devices. Chanda, et al. (2016) designed and implemented a wireless gesture control robot using Arduino ATMEGA32 processor and Android operated application system. The system organizes broadly classified development function into two components. The hardware part consisting of Arduino Microcontroller, the Adafruit motor shield, HC-05 Bluetooth module, and the Android smartphone, and the software part consists of a java-based application run on android. Dey, et al. (2015) designed and developed a three-way control android smartphone based robotic vehicle via Bluetooth. They have developed a three-way control for the robotic vehicle in which we have used Bluetooth communication to interface the microcontroller and the inbuilt sensors in the Android smartphone. According to commands received from the android phone, the kinematics of the robot is controlled. The developed robotic vehicle can be used for numerous applications in the future especially in the field of surveillance and security. Patchava, et al. (2015) studied and explained a smart home automation technique with Raspberry Pi using IoT technology. This proposed system for the Smart Home automation technique with Raspberry Pi using IoT and it developed by integrating cameras and motion sensors into a web application. To design this system, they are using a Raspberry Pi module with computer vision techniques.

\section{IOT SERVICE AND APPLICATIONS}

The IoT brings the power of the internet, data processing and analytics to the real world of physical objects. For consumers, this means interacting with the global information network without the intermediary of a keyboard, mouse and screen; many of their everyday objects and appliances can take instructions from that network with minimal human intervention. IoT can create a very intelligent environment using smart devices. Paired with mobile applications that enable users to control these devices remotely, this technology is one of the most remarkable technologies of this era. Therefore, in this section, we study and explain useful IoT technology services and different IoT categories like newly named IoT.

\section{IoT Service for Smart Home}

An IoT smart home is a technology that uses internet-connected devices to enable the remote monitoring and management of appliances and systems, such as lighting and heating. IoT smart home systems achieved great popularity in the last decades as they increase the comfort and quality of life. Most IoT smart home systems are controlled by smartphones and microcontrollers (Malche and Maheshwary, 2017). A smartphone application is used to control and monitor home functions using wireless communication techniques. Alaa, et al. (2017) studied and explored the concept of IoT smart home with the integration of IoT services and cloud computing to it, by embedding intelligence into sensors and actuators, networking of smart things using the corresponding technology, facilitating interactions with smart things using cloud computing for easy access in different locations, increasing computation power, storage space and improving data exchange efficiency. Currently, a smart home using IoT and data science lives up to the consumer's expectations and sometimes even exceeds them. Using sensors, devices, appliances, and the whole spaces in your house constantly collects data on how you use them. They learn about your habits and determine consumption patterns using complex algorithms. These insights then help personalize your experience at a granular level (Stojkoska and Trivodaliev, 2017). IoT smart home devices of the new generation use their sensor 
datum to automatically adjust regimes to your routines. They monitor your location in real-time and turn the heating on and off accordingly (Jang, et al. 2017). The best part is that you really do not have to do anything. Smart thermostats rely on their algorithms to personalize the use of heating to your needs and save you good money on reduced energy use (Al-Ali, et al. 2017).

\section{IoT Service for Healthcare}

IoT for healthcare can also be used for research purposes. IoT can automate patient care workflow with the help of healthcare mobility solutions and other new technologies, and next-gen healthcare facilities. Because IoT enables us to collect a massive amount of data about the patient's illness, which would have taken many years if we collected it manually, it is a very useful technology. IoT-enabled devices have made remote monitoring in the healthcare sector possible, unleashing the potential to keep patients safe and healthy, and empowering physicians to deliver superlative care. It has also increased patient engagement and satisfaction as interactions with doctors have become easier and more efficient. Furthermore, remote monitoring of patient's health helps in reducing the length of hospital stay and prevents re-admissions. IoT also has a major impact on reducing healthcare costs significantly and improving treatment outcomes (Elhoseny, et al. 2018; Yeh, 2016). IoT is undoubtedly transforming the healthcare industry by redefining the space of devices and people interaction in delivering healthcare solutions. IoT has applications in healthcare that benefit patients, families, physicians, hospitals, and insurance companies (Tyagi, et al. 2016). An aspect of IoT for patients, devices in the form of wearables like fitness bands and other wirelessly connected devices like blood pressure and heart rate monitoring cuffs, glucometer, etc. give patients access to personalized attention. These devices can be tuned to remind calorie count, exercise check, appointments, blood pressure variations and much more (Kodali, et al. 2015). IoT has changed people's lives, especially elderly patients, by enabling constant tracking of health conditions. This has a major impact on people living alone and their families. On any disturbance or changes in the routine activities of a person, an alert mechanism sends signals to family members and concerned health providers (Yeole and Kalbande, 2016). By using wearables and other home monitoring equipment embedded with IoT, physicians can keep track of patients' health more effectively. They can track patients' adherence to treatment plans or any need for immediate medical attention. IoT enables healthcare professionals to be more watchful and connect with the patients proactively. Data collected from IoT devices can help physicians identify the best treatment process for patients and reach the expected outcomes (Babu, et al. 2016). Apart from monitoring patients' health, there are many other areas where IoT devices are very useful in hospitals. IoT devices tagged with sensors are used for tracking real time locations of medical equipment like wheelchairs, defibrillators, nebulizers, oxygen pumps and other monitoring equipment. Deployment of the medical staff at different locations can also be analyzed real time (Kulkarni and Sathe, 2014). Particularly, the spread of infections is a major concern for patients in hospitals as recently COVID19 pandemic. IoT-enabled hygiene monitoring devices help in preventing patients from getting infected. IoT devices also help in asset management like pharmacy inventory control, and environmental monitoring, for instance, checking refrigerator temperature, and humidity and temperature control.

\section{IoT Applications}

IoT devices are used in a variety of different fields for a board range of functions. Recently, several IoT devices are used in a variety of different fields for a board range of functions. According to a few kinds of research, it generates and uses a new name as an IoT application. The Industrial Internet of Things (IIoT) refers to the extension and use of the internet of things (IoT) in industrial sectors and applications. With a strong focus on machine-to-machine (M2M) communication, big data, and machine learning, the IIoT enables industries and enterprises to have better efficiency and reliability in their operations. The IIoT encompasses industrial applications, including robotics, medical devices, and software-defined production processes (Hossain and Muhammad, 2016). The Internet of Medical Things (IoMT) is an amalgamation of medical devices and applications that can connect to health care information technology systems using networking technologies. It can reduce unnecessary hospital visits and the burden on health care systems by connecting patients to their physicians and allowing the transfer of medical data over a secure network (Joyia, et al. 2017). Atzori, et al. (2012) are introduced "SIoT" concept of Social IoT in their research paper named Social Internet of Things (SIoT), has the potential to support novel applications and networking services for the IoT in more active and client-useful ways. It is the convergence of the "Internet of Things" and the "Social Networks" worlds is possible, or even advisable, is gaining momentum. This is due to the growing awareness that a "Social Internet of Things" paradigm would carry many desirable implications into a future world populated by intelligent objects permeating the everyday life of human beings. 


\section{DEVELOPMENT METHOD OF INTEGRATED IOT KNOWLEDGE}

As the IoT continues its expansion into robotics, homes, hospitals, healthcare, businesses, government, global culture, and every industry, the impact for computer science (or the information and engineering) educators is being amplified. Smart devices are everywhere, affecting virtually every aspect of daily life, such as social life, healthcare, entertainment, manufacturing, and education. In this study, we conceptually propose IoT framework describe including the five layers of the IoT technology stack (Figure 2). Learning and managing the complexity of the five layers of technology (device hardware, device software, communications, cloud platform, and cloud applications) can be daunting. In this section, we assume and explain that select one solution for remote control IoT based on the Android mobile application case.

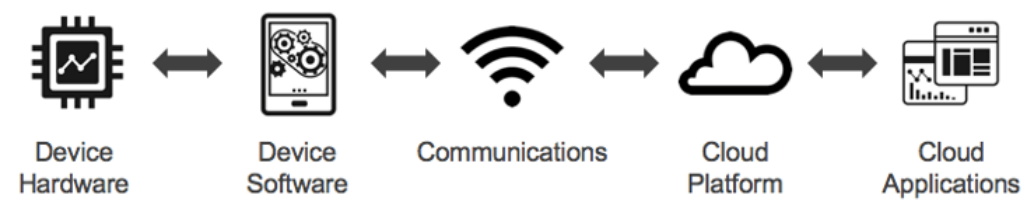

Figure 2. IoT technology five layers stack

However, when we teaching IoT, requires addressing several challenging questions, such as: How will we integrate IoT concepts and technologies into existing curricula? How will we handle the mix of software and hardware topics that most IoT classes or projects involve? How will we deal with the legal, moral, social, and ethical issues? How will we choose from the growing number of IoT service platforms and industry standards? The basic research questions we address in this study are:

Q1. What is the most efficient teaching to integrate the IoT development process of the IoT project?

Q2. What are the most efficient criteria for making decisions related to IoT project development in computer science classes?

The hardware utilized in IoT systems includes devices for a remote dashboard, devices for control, servers, a routing or bridge device, and sensors. These devices manage key tasks and functions such as system activation, action specifications, security, communication, and detection to support-specific goals and actions. When a course involves IoT projects, the instructor needs to choose one or more hardware platforms (e.g., Raspberry Pi, Arduino, ESP8266, NXP Pico, micro: bit, LightBlue Bean, etc.), one or more software platforms (e.g., C, Java, Python, Android, etc.), and one or more network services (e.g., Ethernet, ZigBee, Bluetooth, Wi-Fi, etc.). In many cases, IoT knowledge decisions about hardware and software are interrelated.

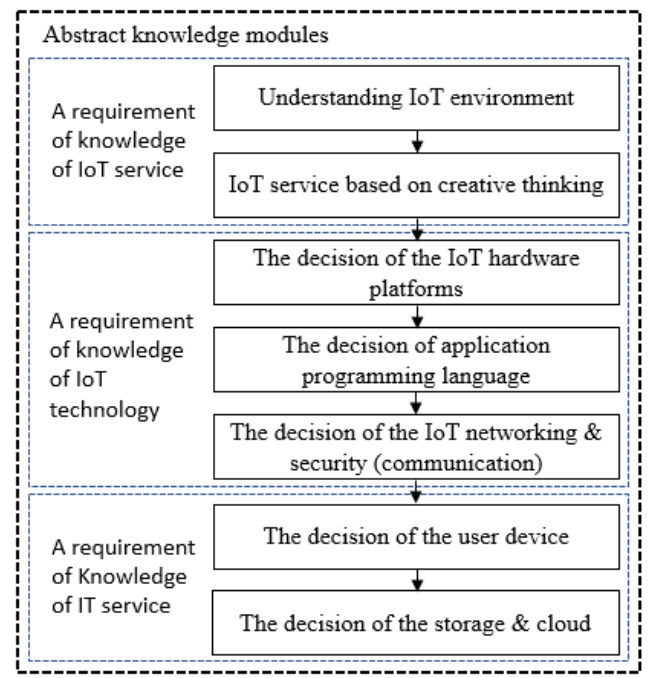

Figure 3. Knowledge modules of integrating IoT development process 
Figure 3 explains how to integrate the IoT development process for IoT projects. Basically, this integrated group requires three different knowledge modules. First, IoT learners need to understand the IoT environment and service. In this step, IoT learners can create a project subject or title by creative thinking for their IoT projects. Second, for the project hands-on learning, IoT learners decide IoT hardware platforms, programming language, and communication methods, etc. The IoT project's successful results depend upon the preparation of the IoT integrated knowledge based IoT learners. For example, IoT learners decide to use the Arduino hardware platform and C or python language for the IoT development process. In this step decide the last requirement of knowledge of IT service. Perhaps, the user device could use a smartphone or tablet.

\section{IoT Hardware Platforms}

IoT hardware includes a wide range of devices such as devices for routing, bridges, sensors, etc. These IoT devices manage key tasks and functions such as system activation, security, action specifications, communication, and detection of support-specific goals and actions. IoT hardware components can vary from low-power boards; singleboard processors like the Arduino Uno which are basically smaller boards that are plugged into mainboards to improve and increase its functionality by bringing out specific functions or features (such as GPS, light and heat sensors, or interactive displays). A developer specifies a board's input and output, then creates a circuit design to illustrate the interaction of these inputs and outputs (Figure 4, (a)). Arduino was initially developed for the maker community but has gained in popularity among educators at all levels. Thanks to software libraries that simplify the writing of code, students are able to develop challenging projects with limited or no knowledge of electronics. Another well-known IoT platform is Raspberry Pi 2, which is a very affordable and tiny computer that can incorporate an entire web server. Often called "RasPi," it has enough processing power and memory to run Windows 10 on it as well as IoT Core. Raspberry Pi exhibits great processing capabilities, especially when using the Python programming language (Figure 4, (b)). The Raspberry Pi has more computational power and might be an appropriate choice for handling elaborate data, executing complex tasks, or extending connectivity. BeagleBoard is a single-board computer with a Linux-based OS that uses an ARM processor, capable of more powerful processing than Raspberry Pi. Tech giant Intel's Galileo and Edison boards are other options, both great for larger-scale production, and Qualcomm has manufactured an array of enterprise-level IoT technology for cars and cameras to healthcare (Figure 4, (c)). There are many versions of the microcontroller hardware platforms, with new versions being released frequently. Figure 4 shows the three most popular prototyping microcontroller hardware platforms are Arduino, Raspberry Pi and BeagleBoard.

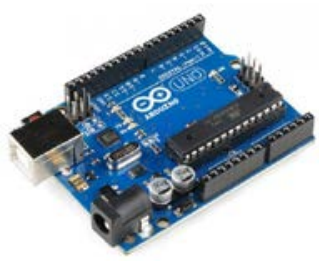

(a) Arduino UNO

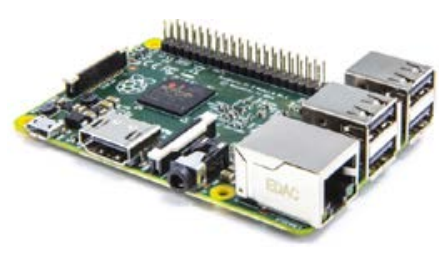

(b) Raspberry Pi 2

Figure 4. IoT Microcontroller Hardware

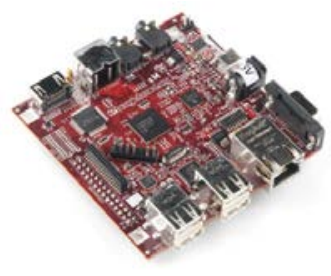

(c) BeagleBoard

The most important hardware in IoT might be its sensors. These devices consist of energy modules, power management modules, RF modules, and sensing modules. RF modules manage communications through their signal processing, WiFi, ZigBee, Bluetooth, radio transceiver, duplexer, and BAW (Porkodi and Bhuvaneswari, 2014). Among one of the most extensively made uses of wireless technologies of the short-range is Bluetooth. We can quickly obtain Bluetooth apps that provide you with wearable technology for pairing with wise gadgets (Harris, et al. 2016).

\section{IoT Programming Languages}

The software and the programming languages on which IoT works uses very common programming languages that programmers use and already know. Which language should be chosen for efficient learning for major or non-major students? According to research papers (Singh \& Kapoor, 2017), developers are using several different programming languages for IoT such as structural programming, object-oriented programming, and visual program (or block program). Since 2015, the Eclipse Foundation has been surveying the developers working in IoT development companies worldwide to understand the overall landscape, challenges, and trends in this field. The latest IoT developer 
survey 2018 was conducted between January and March 2018 in which 502 individuals participated. According to this survey, developers are using several different programming languages for IoT such as C, C++, Java, JavaScript, Python, and PHP to name a few. Because embedded systems have less storage and processing power, their language needs are different. The most used operating systems for such embedded systems are Linux or UNIX-like OSs like Ubuntu Core or Android. IoT software encompasses a wide range of software and programming languages from general-purpose languages like C++ and Java to embedded-specific choices like Google’s Go language or Parasail. More commonly, IoT systems are a distributed application leveraging a variety of web services. But it is true that majors or non-major developers can find it difficult to learn IoT from several different programming languages.

However, many researchers and students develop and using for IoT with block-based programming. Zubrycki, et al. (2017) presented the implementation of a graphical environment for programming a set of internet-of-things (IoT) devices and robots for therapists of autism. It was based on the Robot Operating System (ROS) and Snap and is called Robokol. This research paper implemented the elements and functionality of the system described browser-based graphical programming interface Snap, to integrate it with ROS (Robot Operating System). Robokol used as a way to prototype an interaction between a child and a device was tested in several settings. De Lucar, et al. (2018) developed and presented a visual programming language and its development environment for programming IoT and robotics devices. ASU VIPLE supports LEGO EV3 and all IoT devices based on an open architecture. MIT App Inventor is a web application integrated development environment originally provided by Google and now maintained by the Massachusetts Institute of Technology. The App Inventor development environment is supported for Mac OS X, GNU/Linux, and Windows operating systems, and several popular Android phone models. Applications created with App Inventor can be installed on any Android phone. Besides MIT App Inventor, there are other block-based programming environments supporting Arduino (e.g., Scratch, Snap4Arduino, Microblocks, BlocklyDuino) with different block designs (Xi and Patton, 2018). MIT App Inventor is an online platform designed to teach computational thinking concepts through the development of mobile applications. Students create applications by dragging and dropping components into a design view and using a visual blocks language to program application behavior (Patton, et al. 2019). According to the Abdullaha and Varolb (2019) research, they implemented an efficient Android Software Development Using MIT App Inventor 2 for Bluetooth-Based Smart Home. Figure 5 explains how a learner can perform IoT development in App Inventor online platform. Therefore, in this paper, we propose a block-based programming approach using App Inventor to enable novices to be able to build mobile apps integrated with IoT technology.

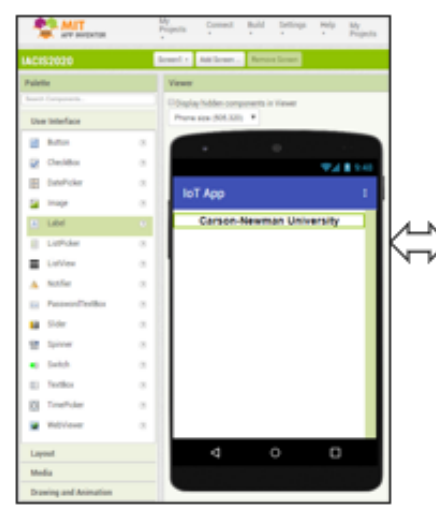

App Inventor Designer Editor

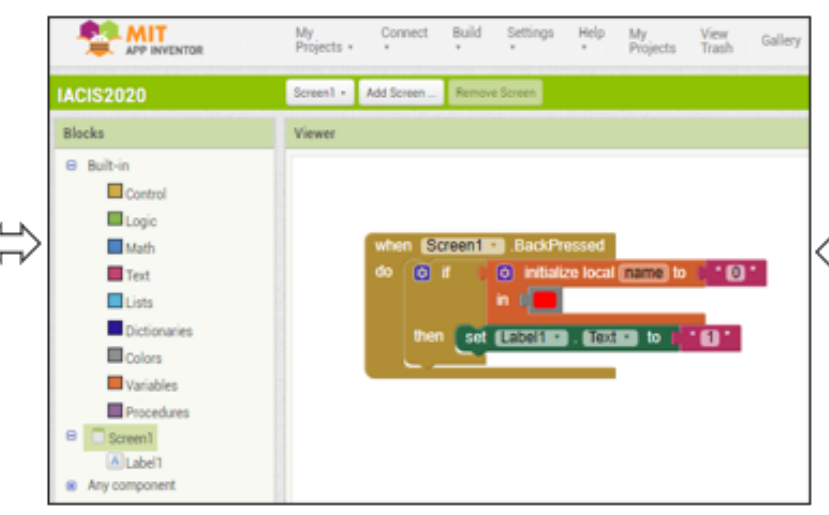

App Inventor Blocks Editor

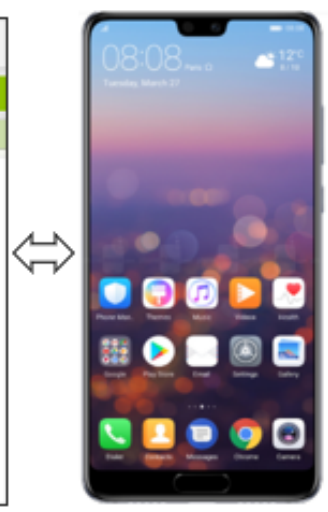

Mobile Device

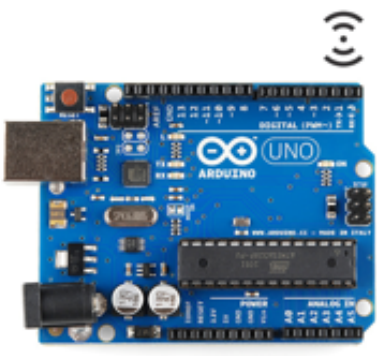

IoT Microcontroller

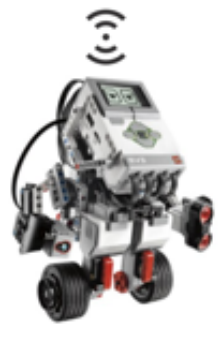

IoT Robot controller

Figure 5. Integrated IoT in the App development environment 


\section{AN EXAMPLE OF PROJECT BY INTEGRATE IOT KNOWLEDGE}

In this section, we explain the experiment on the efficient education efforts, applied integrated IoT knowledge, and mobile application programming presented in this paper. The Internet of Things can be integrated into lesson plans and activities in the classroom at all levels. This paper presents two experiences acquired in teaching for the Internet of Things as a project of the course named "Senior Capstone Project" or "Special Topic - Robotics Programming" to computer science students. The main goal was to motivate students to become more prepared for future jobs in computer science, IT field and interested in STEM (Science, Technology, Engineering, and Mathematics) or STEAM (STE+Arts+Math). Figures 6 and 7 explain the study of students' integrated IoT projects. In the first step, for the knowledge of IoT service, we suggested a few integrated IoT knowledge solutions to students, after understanding the IoT environment. In this step, students create their creative ideas for IoT projects by brainstorming. In the second step, for the knowledge of IoT technology, we suggested a few IoT hardware platforms, application programming languages and IoT communication after learning technology concepts. In this part, we need to consider the cost issues and the lab exercise environment. In the last step, for the knowledge of IT service, we suggested a few user devices and cloud solutions to students, after understanding the IT service environment. We also need to consider the cost issues and the lab environment in this area. For example, if we want to use a smartphone device, it is very expensive for students to purchase. Figure 7 shows an integrated IoT project based on Arduino for the senior capstone project. This project idea basically controls the LED display by the mobile device for smart pill dispenser. Therefore, students decided to use Arduino hardware platform, App Inventor block program, HC-06 Bluetooth, and Samsung Galaxy Android device for making their IoT project. As shown in Figure 8, this is another integrated IoT project based on the Lego EV3 robot for a robot programming class. This project idea basically studies and controlled robot wheel motors and sensors by the mobile device for remote control robotics. Accordingly, students decided to use the EV3 Mindstorm hardware platform, App Inventor block program, EV3 Bluetooth, and Samsung Galaxy Android device for making their IoT project.

\begin{tabular}{|c|c|c|c|}
\hline $\begin{array}{c}\text { The decision of the IoT } \\
\text { hardware platforms }\end{array}$ & $\begin{array}{c}\text { The decision of application } \\
\text { programming language }\end{array}$ & $\begin{array}{c}\text { The decision of the IoT } \\
\text { networking } \\
\text { (communication module) }\end{array}$ & $\begin{array}{c}\text { The decision of the User } \\
\text { Device } \\
\text { (mobile module) }\end{array}$ \\
\hline Arduino UNO & MIT App Inventor & Bluetooth HC-06 & Samsung Galaxy Android \\
\hline & &
\end{tabular}

Figure 6. Integrated IoT Project Case Study I

\begin{tabular}{|c|c|c|c|}
\hline $\begin{array}{c}\text { The decision of the IoT } \\
\text { hardware platforms }\end{array}$ & $\begin{array}{c}\text { The decision of application } \\
\text { programming language }\end{array}$ & $\begin{array}{c}\text { The decision of the IoT } \\
\text { networking } \\
\text { (communication module) }\end{array}$ & $\begin{array}{c}\text { The decision of the User } \\
\text { Device } \\
\text { (mobile module) }\end{array}$ \\
\hline Lego EV3 robot & MIT App Inventor & EV3 Bluetooth & Samsung Galaxy Android \\
\hline & \begin{tabular}{c} 
\\
\hline
\end{tabular} & \\
\hline
\end{tabular}

Figure 7. Integrated IoT Project Case Study II

After IoT introductory lessons, students divided into a team or individually and chose a topic they would study in detail, research, and then present to others and prepare questions for discussion. In the presented case study, familiarizing students with the Internet of Things concept, its possibilities, and applications, consisted of theoretical 
and practical lessons. The teaching methods that emphasized collaborative and active learning, creative problemsolving and critical thinking were used. Consequently, students can quickly learn and develop efficiently through the integrated IoT knowledge.

\section{CONCLUSIONS}

According to our review, it is difficult to teach IoT technology to computer science or information learners because of IoT requires a huge service concept and technology knowledge. IoT is an umbrella term with many use cases, technologies, standards, and applications. However, a lot of students and developers want to learn IoT. We focused and studied fundamental questions, what is an efficient way to learn the Internet of Things in computer science or information major? Therefore, in this study, we discussed a method of efficient developing IoT applications using integrated physical devices and mobile-based programming knowledge for computer science major colleges. In addition, we conceptualized the integrated IoT development knowledge process for IoT courses. According to this IoT development knowledge process, we can simply be understanding knowledge of IoT technology. The integrated IoT development knowledge process can improve the IoT project process and learner IoT skills. For the validation, we proposed two case studies for an integrated IoT project. The next step for our research is to further investigate on learner's IoT learning satisfaction result and find some improvement for the integrated IoT development knowledge process.

\section{REFERENCES}

Atzori, L., Iera, A., \& Morabito, G. (2010). The internet of things: A survey. Computer networks, 54(15), 2787-2805.

Atzori, L., Iera, A., Morabito, G., \& Nitti, M. (2012). The social internet of things (siot)-when social networks meet the internet of things: Concept, architecture and network characterization. Computer networks, 56(16), 35943608.

Alaa, M., Zaidan, A. A., Zaidan, B. B., Talal, M., \& Kiah, M. L. M. (2017). A review of smart home applications based on Internet of Things. Journal of Network and Computer Applications, 97, 48-65.

Ali, F. (2015, October). Teaching the internet of things concepts. In Proceedings of the WESE'15: Workshop on Embedded and Cyber-Physical Systems Education (pp. 1-6).

Al-Ali, A. R., Zualkernan, I. A., Rashid, M., Gupta, R., \& AliKarar, M. (2017). A smart home energy management system using IoT and big data analytics approach. IEEE Transactions on Consumer Electronics, 63(4), 426434.

Abdullaha, A. S., \& Varolb, A. (2019) Smart Home Automation using Internet of Things (IOT). International Turkish World Engineering and Science Congress (pp. 261-267).

Arasteh, H., Hosseinnezhad, V., Loia, V., Tommasetti, A., Troisi, O., Shafie-khah, M., \& Siano, P. (2016, June). Iotbased smart cities: a survey. In 2016 IEEE 16th International Conference on Environment and Electrical Engineering (EEEIC) (pp. 1-6). IEEE.

Babu, B. S., Srikanth, K., Ramanjaneyulu, T., \& Narayana, I. L. (2016). IoT for healthcare. International Journal of Science and Research, 5(2), 322-326.

Chanda, P., Mukherjee, P., \& SubrataModak, A. (2016). Gesture Controlled Robot using Arduino and Android. International Journal, 6(6).

De Luca, G., Li, Z., Mian, S., \& Chen, Y. (2018). Visual programming language environment for different IoT and robotics platforms in computer science education. CAAI Transactions on Intelligence Technology, 3(2), 119130. 
De La Concepcion, A. R., Stefanelli, R., \& Trinchero, D. (2014, October). A wireless sensor network platform optimized for assisted sustainable agriculture. In IEEE global humanitarian technology conference (GHTC 2014) (pp. 159-165). IEEE.

Deshpande, A., Pitale, P., \& Sanap, S. (2016). Industrial automation using Internet of Things (IOT). International Journal of Advanced Research in Computer Engineering \& Technology (IJARCET), 5(2), 266-269.

Dey, A., Pal, A., Nandi, S., \& Roy, L. (2015). Three way controlled android Smartphone based robotic vehicle via Bluetooth. International Journal of Advanced Research in Computer and Communication Engineering, 4(9), 212-216.

Díaz Pardo de Vera, D., Sigüenza Izquierdo, Á., Bernat Vercher, J., \& Hernández Gómez, L. A. (2014). A Ubiquitous sensor network platform for integrating smart devices into the semantic sensor web. Sensors, 14(6), 1072510752.

Elhoseny, M., Ramírez-González, G., Abu-Elnasr, O. M., Shawkat, S. A., Arunkumar, N., \& Farouk, A. (2018). Secure medical data transmission model for IoT-based healthcare systems. Ieee Access, 6, 20596-20608.

Fernández-Caramés, T. M., \& Fraga-Lamas, P. (2018). Towards the Internet of smart clothing: A review on IoT wearables and garments for creating intelligent connected e-textiles. Electronics, 7(12), 405.

Harris III, A. F., Khanna, V., Tuncay, G., Want, R., \& Kravets, R. (2016). Bluetooth low energy in dense IoT environments. IEEE Communications Magazine, 54(12), 30-36.

Hossain, M. S., \& Muhammad, G. (2016). Cloud-assisted industrial internet of things (iiot)-enabled framework for health monitoring. Computer Networks, 101, 192-202.

Jang, W., Chhabra, A., \& Prasad, A. (2017, November). Enabling multi-user controls in smart home devices. In Proceedings of the 2017 Workshop on Internet of Things Security and Privacy (pp. 49-54).

Joyia, G. J., Liaqat, R. M., Farooq, A., \& Rehman, S. (2017). Internet of Medical Things (IOMT): applications, benefits and future challenges in healthcare domain. J Commun, 12(4), 240-7.

Krasniqi, X., \& Hajrizi, E. (2016). Use of IoT technology to drive the automotive industry from connected to full autonomous vehicles. IFAC-PapersOnLine, 49(29), 269-274.

Koreshoff, T. L., Robertson, T., \& Leong, T. W. (2013, November). Internet of things: a review of literature and products. In Proceedings of the 25th Australian Computer-Human Interaction Conference: Augmentation, Application, Innovation, Collaboration (pp. 335-344).

Kodali, R. K., Swamy, G., \& Lakshmi, B. (2015, December). An implementation of IoT for healthcare. In 2015 IEEE Recent Advances in Intelligent Computational Systems (RAICS) (pp. 411-416). IEEE.

Kulkarni, A., \& Sathe, S. (2014). Healthcare applications of the Internet of Things: A Review. International Journal of Computer Science and Information Technologies, 5(5), 6229-6232.

Monnier, O. (2013). A smarter grid with the Internet of Things. Texas Instruments, 1-11. 
Malche, T., \& Maheshwary, P. (2017, February). Internet of Things (IoT) for building smart home system. In 2017 International Conference on I-SMAC (IoT in Social, Mobile, Analytics and Cloud)(I-SMAC) (pp. 65-70). IEEE.

Patton, E. W., Tissenbaum, M., \& Harunani, F. (2019). MIT App Inventor: Objectives, Design, and Development. In Computational Thinking Education (pp. 31-49). Springer, Singapore.

Pahuja, R., \& Kumar, N. (2014). Android mobile phone controlled bluetooth robot using 8051 microcontroller. International Journal of Scientific Engineering and Research, 2(7), 14-17.

Patchava, V., Kandala, H. B., \& Babu, P. R. (2015, December). A smart home automation technique with raspberry pi using iot. In 2015 International conference on smart sensors and systems (IC-SSS) (pp. 1-4). IEEE.

Porkodi, R., \& Bhuvaneswari, V. (2014, March). The internet of things (IOT) applications and communication enabling technology standards: An overview. In 2014 International conference on intelligent computing applications (pp. 324-329). IEEE.

Sivaraman, V., Gharakheili, H. H., Vishwanath, A., Boreli, R., \& Mehani, O. (2015, October). Network-level security and privacy control for smart-home IoT devices. In 2015 IEEE 11th International Conference on Wireless and Mobile Computing, Networking and Communications (WiMob) (pp. 163-167). IEEE.

Singh, K. J., \& Kapoor, D. S. (2017). Create Your Own Internet of Things: A survey of IoT platforms. IEEE Consumer Electronics Magazine, 6(2), 57-68.

Stojkoska, B. L. R., \& Trivodaliev, K. V. (2017). A review of Internet of Things for smart home: Challenges and solutions. Journal of Cleaner Production, 140, 1454-1464.

Tan, P., Wu, H., Li, P., \& Xu, H. (2018). Teaching management system with applications of RFID and IoT technology. Education Sciences, 8(1), 26.

Tyagi, S., Agarwal, A., \& Maheshwari, P. (2016, January). A conceptual framework for IoT-based healthcare system using cloud computing. In 2016 6th International Conference-Cloud System and Big Data Engineering (Confluence) (pp. 503-507). IEEE.

Xi, W., \& Patton, E. W. (2018). Block-Based Approaches to Internet of Things in MIT App Inventor.

Yeole, A. S., \& Kalbande, D. R. (2016, March). Use of internet of things (iot) in healthcare: A survey. In Proceedings of the ACM Symposium on Women in Research 2016 (pp. 71-76).

Yeh, K. H. (2016). A secure IoT-based healthcare system with body sensor networks. IEEE Access, 4, 10288-10299.

Yeole, A. R., Bramhankar, S. M., Wani, M. D., \& Mahajan, M. P. (2015). Smart phone controlled robot using ATMEGA328 microcontroller. International Journal of Innovative Research in Computer and Communication Engineering, 3(1), 352-356.

Zubrycki, I., Kolesiński, M., \& Granosik, G. (2017, June). Graphical programming interface for enabling nontechnical professionals to program robots and internet-of-things devices. In International Work-Conference on Artificial Neural Networks (pp. 620-631). Springer, Cham. 